\title{
On the use of multispectral conjunctival vasculature as a soft biometric*
}

\author{
Simona Crihalmeanu \\ West Virginia University \\ Morgantown WV 26506, USA \\ Simona.Crihalmeanu@mail.wvu.edu
}

\author{
Arun Ross \\ West Virginia University \\ Morgantown WV 26506, USA \\ Arun.Ross@mail.wvu.edu
}

\begin{abstract}
Ocular biometrics has made significant progress over the past decade primarily due to advances in iris recognition. Initial research in the field of iris recognition focused on the acquisition and processing of frontal irides which may require considerable subject cooperation. However, when the iris is off-angle with respect to the acquisition device, the sclera (the white part of the eye) is exposed. The sclera is covered by a thin transparent layer called conjunctiva. Both the episclera and conjunctiva contain blood vessels that are observable from the outside. In this work, these blood vessels are referred to as conjunctival vasculature. Iris patterns are better observed in the near infrared spectrum while conjunctival vasculature is better seen in the visible spectrum. Therefore, multispectral (i.e., color-infrared) images of the eye are acquired to allow for the combination of the iris biometric with the conjunctival vasculature. The paper focuses on conjunctival vasculature enhancement, registration and matching. Initial results are promising and suggest the need for further investigation of this biometric in a bimodal configuration with iris.
\end{abstract}

\section{Introduction}

Ocular biometrics has made significant progress over the past decade primarily due to advances in iris recognition [9][1]. The iris has been demonstrated to be a reliable biometric with high variability across individuals when imaged in the near-infrared (NIR) spectrum [3]. Initial research focused on the acquisition and processing of frontal iris images. However, when the iris is off-angle with respect to the acquisition device, the sclera (also known as the white of the eye) is exposed. The sclera is the external layer of the eye, and is a firm dense membrane comprising of a white and opaque fibrin connective tissue, organized in many bands of parallel and interlacing fibrous tissue bundles. Its outer

*Thanks to Dr. Reza Derakhshani for the useful discussions and to Peter Hein for assisting us with the data collection. This work was partially funded by the Center for Identification Technology Research (CITeR). surface, called episclera, contains the blood vessels nourishing the sclera. The anterior part of the sclera is covered by the conjunctival membrane, a thin layer that helps lubricate the eye for eyelid closure. The rich vasculature revealed in the episclera and conjunctival membrane is referred to as conjunctival vasculature in this paper. Previous studies [4][2] investigated the feasibility of using the conjunctival vasculature patterns, imaged in the visible spectrum, as a biometric. Iris patterns are better observed in the NIR spectrum while the vasculature patterns are better observed in the visible spectrum (RGB). Therefore, multispectral images of the eye can be potentially used for combining the iris biometric with the conjunctival vasculature for improved recognition. This paper focuses on multispectral conjunctival vasculature enhancement and matching. The conjunctival vasculature is viewed as a soft biometric since it is expected to have limited discrimination capability compared to the iris.

The paper is organized as follows: section 2 describes multispectral data acquisition; section 3 presents the process of image denoising; section 4 describes specular reflection detection and removal; section 5 discusses sclera segmentation; section 6 describes conjunctival vasculature enhancement; section 7 describes the registration of the images; section 8 presents experimental results evaluating the recognition accuracy. The block diagram of the proposed system is shown in Figure 1.

\section{Image acquisition}

\subsection{Multispectral Imaging}

Multispectral imaging captures the image of an object at multiple spectral bands often ranging from the visible spectra to the infra-red spectra. The visible spectral band [5] comprises of three narrow sub-bands called the red, green and blue channels that range from $0.4 \mu \mathrm{m}$ to $0.7 \mu \mathrm{m}$. The infrared spectrum is divided into NIR (near-infrared), MIR (midwave infrared), FIR (far infrared) and thermal bands, ranging from $0.7 \mu \mathrm{m}$ to over $10 \mu \mathrm{m}$. 


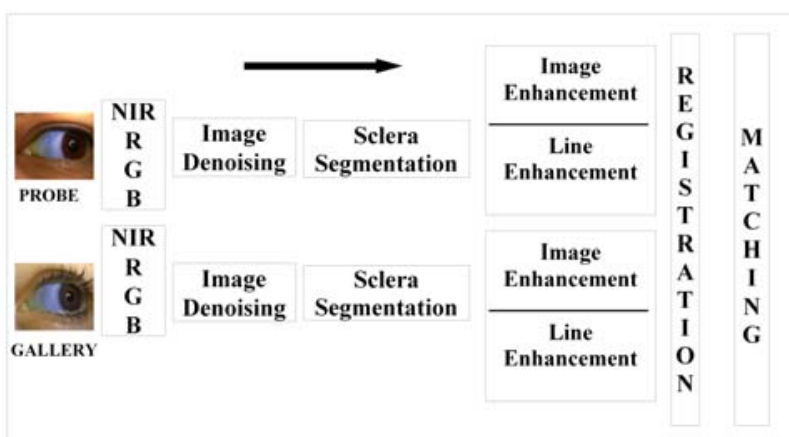

Figure 1. Block diagram showing the pre-processing and matching of multispectral conjunctival vasculature

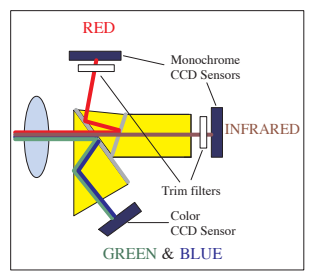

(a)

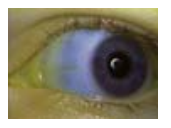

(b)

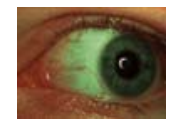

(f)

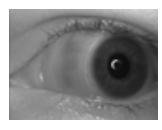

(c)

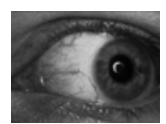

(g)

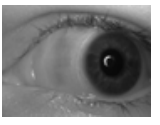

(d)

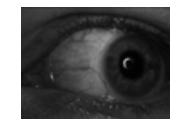

(h)

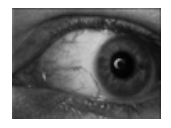

(e)

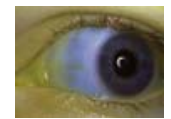

(i)
Figure 2. a) The DuncanTech MS3100 camera: CIR/RGB Spectral Configuration (Adapted from Hi-Tech Electronics: www.hitech.com.sg). (b) Color-infrared image (NIR-RedBayerPattern). (c) NIR component. (d) Red component. (e) Bayer pattern. (f) RGB image. (g) Green component. (h) Blue component. (i) Composite image (NIR-Red-Green)

\subsection{Multispectral acquisition system}

Images of the eye are collected using the Redlake (DuncanTech) MS3100 multispectral camera. ${ }^{1}$ The camera has three array sensors. Between the lenses and the sensors there is a color-separating prism to split the ingoing broadband light into three optical channels. Figure 2(a) displays the configuration of the multispectral camera.

The camera acquires imagery of four spectral bands from a three channel optical system. As specified by the sensor manufacturer, the center wavelength of each spectral band is as follows: blue - $460 \mathrm{~nm}$, green - $540 \mathrm{~nm}$, red - $660 \mathrm{~nm}$ and NIR - $800 \mathrm{~nm}$. The CIR (Color InfraRed)/RGB configuration outputs three channels represented as a $2 \mathrm{D}$ matrix of pixels that are stacked on top of each other along the $3 \mathrm{rd} \mathrm{di}-$

\footnotetext{
${ }^{1} \mathrm{Hi}-\mathrm{Tech}$ Electronics, Spectral Configuration Guide for DuncanTech 3-CCD Cameras, http://www.hitech.com.sg
}

mension; the three channels correspond to the near-infrared (NIR) component, red component, and a Bayer mosaic-like pattern in which red pixels on the color array are ignored. Figure 2(b)-(i) shows an example of a CIR image along with its components. The first channel - the NIR component - is stored as a separate image. The second channel - the red component - is stored as the red component of the RGB image. The green and blue components are obtained from the third channel of the CIR/RGB configuration through a Bayer pattern demosaicing algorithm. The system used to collect the multispectral images is composed of an ophthalmologist's slit-lamp mount and a light source. The mount consists of a rest chin to position the head and a mobile arm to which the multispectral camera is attached so that it can be easily manipulated to focus on the white of the eye, while the person is gazing to the left or to the right. The light source illuminates the eye using a spectral range from 350 $\mathrm{nm}$ to $1700 \mathrm{~nm}$, and is projected onto the eye via an optic fiber guide with a ring light attached to its end. Because of the reflective qualities of the eyeball, pointing a light source directly at the subject's eye creates a glare on the sclera. The issue is resolved by directing the light source such that the incoming rays to the eyeball are approximately perpendicular to the pupil region. This is not always possible due to subtle movements of the eyeball. Thus, glare is not always contained within the pupil region and may overlap with the iris.

The multispectral camera generates images of size 1040x1392x3 pixels from which the first 17 columns are removed due to artifacts. The final size of the images is, therefore, $1035 \times 1373 \times 3$. Videos of the right and left eye are captured from 49 subjects, with each eye gazing to the right or to the left. 8 images per eye per gaze direction is selected from the video. The total number of images is 1,536 . For two subjects, only data from the right eye was collected due to medical issues. Working with images from the same video allows us to bypass some of the challenges encountered by Crihalmeanu et al. [2] primarily due to viewing angle. The process of frame selection ensures that there is no remarkable change in pose. Our multispectral collection contains images of the eye with different iris colors. Based on the Martin-Schultz scale ${ }^{2}$ often used in physical anthropology, the eyes are classified as light eyes (blue, green gray), mixed eyes (blue, gray or green with brown pigment) and dark eyes (brown, dark brown, almost black). In our work, we consider two categories: light eyes (light and mixed eyes from Martin-Schultz scale) and dark eyes. Examples of the acquired images are displayed in Figure 3.

\footnotetext{
${ }^{2}$ http://wapedia.mobi/en/Eye_color
} 

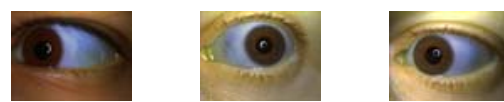

Figure 3. Example of Color-Infrared (CIR) images

\subsection{From Bayer mosaic pattern to RGB}

The Bayer-like pattern is due to the placement of a grid of tiny color filters on the face of the CCD sensor array to filter the light so that only one of the colors (red, blue or green) reaches any given pixel. Here, $25 \%$ of the pixels are assigned to blue, $25 \%$ to red and $50 \%$ to green. Blue and green components are obtained from the Bayer mosaic pattern through interpolation ${ }^{3}$. Figure 2(i) shows a NIRRed-Green composite image.

\section{Image denoising}

The red, green, blue and NIR components obtained from the CIR images are in general noisy (Figure 4(a)(c)(e)(g)). The denoising algorithm employed is based on a wavelet transformation. A double-density complex discrete wavelet transform, (DDCDWT) [11] which combines the characteristics and the properties of the double-density discrete wavelet transform (DDDWT) [10] and the dual-tree discrete wavelet transform (DTDWT) [12], is used. The transformation is based on two scaling functions and four distinct wavelets such that one pair of wavelets form an approximate Hilbert transform pair and the other pair of wavelets are offset from one other by one half. It is implemented by applying four 2-D double density discrete wavelet transforms in parallel to the input data with different filter sets for rows and columns, yielding 32 oriented wavelets (Figure 5(a)) along one of six angles at $\pm 15, \pm 45, \pm 75$ degrees. ${ }^{4}$ The method is shift-invariant, possesses improved directional selectivity and is based on FIR perfect reconstruction filter banks as illustrated in Figure 5(b). For all scales and subbands, the magnitudes of the complex wavelet coefficients are processed by soft thresholding that sets the coefficients with values less than a threshold to zero and subtracts the threshold values from the non-zero coefficients. Original and denoised red, green, blue and NIR images are presented in Figure 4. Visual differences are not pronounced due to image rescaling.

\section{Specular reflection}

Specular reflections have to be detected and removed for a better segmentation of the sclera (described in Section 5). As stated in Section 2.2, the data acquisition system uses a light source to illuminate the eye region. The light di-

\footnotetext{
${ }^{3}$ RGB "Bayer" Color and MicroLenses, http://www.siliconimaging.com/RGB Bayer.htm

${ }^{4}$ http://taco.poly.edu/selesi/DoubleSoftware/index.html
}

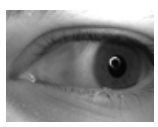

(a)

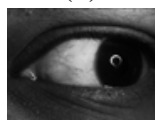

(e)

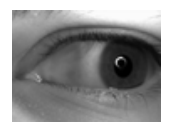

(b)

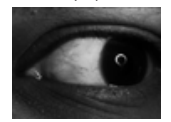

(f)

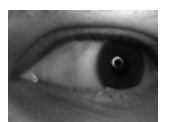

(c)

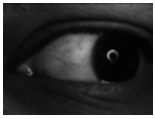

$(\mathrm{g})$

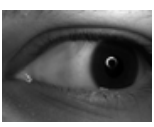

(d)

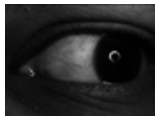

(h)
Figure 4. Denoising with Double Density Complex Discrete Wavelet Transform. a) Original NIR. b) Denoised NIR. c) Original red component. d) Denoised red component. e) Original green component. f) Denoised green component. g) Original blue component. h) Denoised blue component. Visual differences are not pronounced due to image rescaling

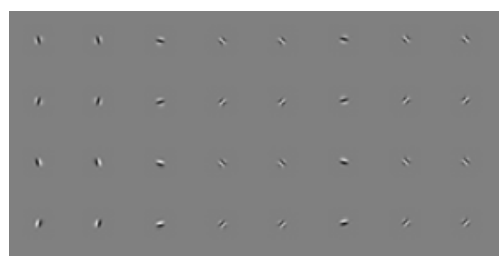

(a)

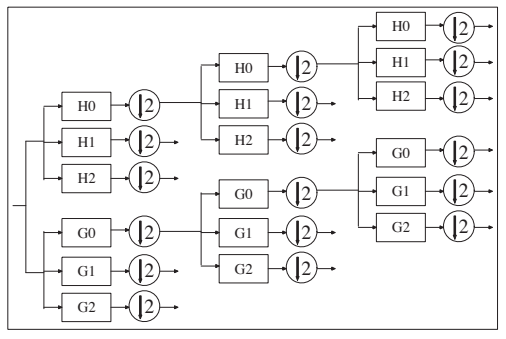

(b)

Figure 5. (a) Plot of Complex 2-D Double-Density Dual-Tree Wavelets. (b) Iterated filterbank for the Double-Density Complex Discrete Wavelet Transform [12]

rected to the eyeball generates specular reflection that has a ring-like shape, caused by the shape of the source of illumination, and highlights, due to the humidity of the eye and the curved shape of the eyeball. Both are detected and removed by a fast inpainting algorithm. In some images, the ring-like shape may be an incomplete circle, ellipse, or an arbitrary curved shape with a wide range of intensity values. It may be located partially in the iris region, making its detection and removal more difficult especially since the iris texture has to be preserved as much as possible. The specular reflections are detected using different threshold values for each component: 0.60 for NIR, 0.50 for red and 0.80 for green. Only regions with less then 3500 pixels in size are labeled as specular reflection and inpainted. In digital inpainting, the information from the boundary of the region to be inpainted is propagated smoothly inside the region. The value to be inpainted at a pixel is calculated using a 
PDE equation ${ }^{5}$ in which partial derivatives are replaced by finite differences between the pixel and its eight neighbors.

\section{Sclera region segmentation}

When the entire image of the eye is used for enhancing the conjunctival vasculature, it is difficult to distinguish between the different types of lines that appear in it: wrinkles, crows feet, eyelashes, blood vessels. Therefore, a good segmentation of the sclera region that clearly exhibits the blood vessels is necessary. The method employed to segment the sclera region is inspired by the work done in the processing of LandSat imagery (Land + Satellite) [13]. Since water absorbs NIR light, the corresponding regions appear dark in the image. The segmentation is based on the fact that the skin has lesser water content than the sclera, and hence exhibits a higher reflectance in NIR. The algorithm to segment the sclera has three main stages as described below.

\subsection{Coarse sclera segmentation}

1. Compute an index called the normalized sclera in$\operatorname{dex} N S I(x, y)=\frac{N I R(x, y)-G(x, y)}{N I R(x, y)+G(x, y)}$, where $N I R(x, y)$ and $G(x, y)$ are the pixel intensities of the NIR and green components, respectively, at pixel location $(x, y)$. The difference $N I R-G$ is larger for pixels pertaining to the sclera region; it is then normalized to help compensate for the uneven illumination (Figures 6(b)(e) and 7(b)). In some cases, for improving the accuracy of segmentation, the intensity values of the NIR component can be adjusted with a $\gamma$ correction value between 0.65 to 0.9 , such that the mapping to the new intensity values is weighted toward higher output values.

2. Locate sclera by thresholding the NSI image with the threshold value $\eta=0.1$. For the right eye of 4 subjects and the left eye of 2 subjects, the segmentation results were better with $\eta=0.12^{6}$. Figure 6(f) displays the scatter plot of NIR intensity values versus the corresponding green intensity values for all pixels in the image. The pixels above the threshold $(\eta=0.1)$ represent the background region while the rest represent the sclera region. Changing the value of $\eta$ will modify the slope of the boundary line between the pixels of the two segmented regions. The output of the thresholding operation is a binary image. Figures 6(c) and 7(c) display the segmented sclera region as the largest blue region (pixels with value 1 in the binary image). ${ }^{7}$ For dark irides (brown and dark brown), the sclera region precluding the iris is localized (Figure $6(\mathrm{~g})$, referred henceforth as

\footnotetext{
${ }^{5}$ http://www.mathworks.com/matlabcentral/fileexchange/4551

${ }^{6}$ When collecting the data, the ring of light is positioned to the left or right side of the eye to ensure uniform illumination across the eye. This introduces some variability in the lighting across different eyes, and hence the small differences in the threshold values

${ }^{7}$ MathWorks, Image Processing Toolbox, Finding Vegetation in a Multispectral Image, http://www.mathworks.com/products/image/demos.html
}

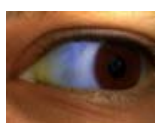

(a)

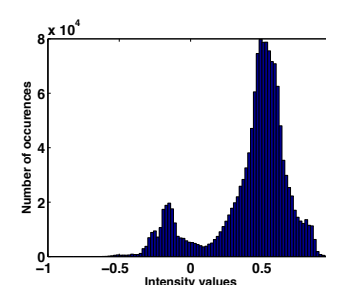

(e)

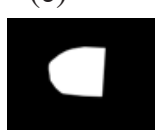

(g)

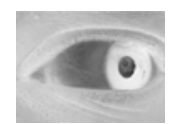

(b)

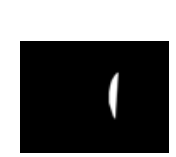

(h)

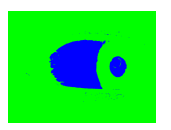

(c)

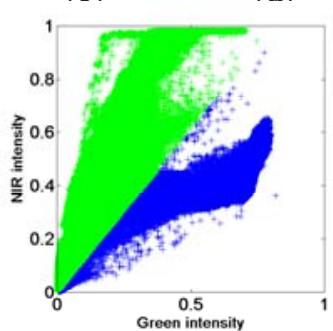

(f)

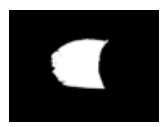

(d)
Figure 6. Sclera region segmentation. Example using a dark color iris. a) Composite image (NIR,R,G). b) Normalized sclera index (NSI). c) Threshold applied to NSI. d) Segmented sclera region $I_{S}$. e) Histogram of the NSI. f) NIR vs green intensity values. g) Convex hull of the sclera region $I_{S C H} . \mathrm{h}$ ) Convex hull of the iris region within the sclera region $I_{I R C H}$. i) Sclera mask. j) Contour of sclera mask imposed on original composite image

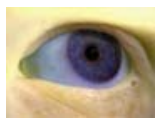

(a)

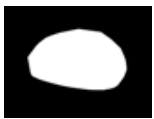

(e)

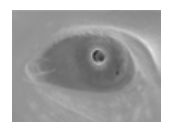

(b)

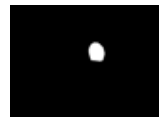

(f)

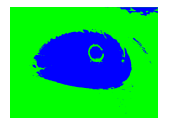

(c)

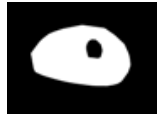

(g)

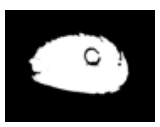

(d)

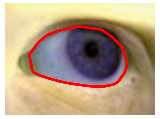

(h)
Figure 7. Sclera region segmentation. Example using a light color iris. a) Composite image (NIR,R,G). b) Normalized sclera index (NSI). c) Threshold applied to NSI. d) Segmented sclera region $I_{S}$. e) Convex hull of the sclera region $I_{S C H}$. f) Convex hull of the iris region within the sclera region $I_{I R C H} . \mathrm{g}$ ) Sclera mask. h) Contour of sclera mask imposed on original composite image

$\left.I_{S}\right)$. Thus, in this case, further segmentation of the sclera and iris is not required. For light irides (blue, hazel, green), regions pertaining to both the sclera and iris are segmented (Figure 7(d), referred henceforth as $I_{S}$ ). Here, further separation of the sclera and iris is needed. The overlapping region between the segmented sclera region as described in this section and the segmented pupil region as will be described in Section 5.2, provides the criteria to differentiate automatically between the two outcomes (corresponding to light or dark iris).

As seen in Figures 6(c) and 7(c), the location of the pupil is also visible either as a blue region that does not overlap the sclera region (in dark irides) or as a green disk within the sclera region (in light irides). Because the extent of overlap 
of the pupil on the segmented sclera depends on the color of the iris, this information can be exploited only if the color of the iris is known in advance. Therefore, in Section 5.2 we present an automatic way of finding the pupil location regardless of the color of the iris.

3. Smooth the contour of the sclera region by finding its convex hull as shown in Figures $6(\mathrm{~g})$ and $7(\mathrm{e}): I_{S C H}=$ ConvexHull $\left(I_{S}\right)$. For dark irides, this operation will include a portion of the iris region that has to be removed; thus, step 4 is needed only for dark color irides. Since the proposed algorithm has no prior information about the color of the iris, it is applied to all images irrespective of eye color.

4. Find the iris region included within the sclera region using the convex hull operator and select the largest connected region. Find its convex hull, $I_{I R C H}$, as shown in Figures 6(h) and 7(f). For light irides this operation will determine the pupil location.

5. Find the sclera mask, shown in Figure 6(i), and Figure $7(\mathrm{~g})$ as the difference $S_{M A S K}=I_{S C H}-I_{I R C H}$. For dark irides, the result of this operation is the sclera mask, and for light irides it is the sclera region without any part of the pupil region. Step 6 is needed for light irides and will have no effect on dark irides.

6. Apply the morphological operation of filling the holes in $S_{M A S K}$ (i.e., the black pixels surrounded by white pixels are set to logical 1).

Figures 6(j) and 7(h) display the contour of the sclera mask when imposed on the original composite image.

\subsection{Pupil region segmentation}

The pupil location is used only to determine if further segmentation of the sclera and iris is needed. Hence, the accurate determination of its boundary is not necessary. In NIR images, the pupil region is characterized by very low intensity values and, by employing a simple threshold, the pupil region is obtained. However, this isolates the eyelashes as well. In order to isolate only the pupil, the following steps are undertaken:

1. Geometrically resize the NIR component by a factor of $1 / 3$ and apply power-law transformation [5] to its pixels: $I_{P L}=c * I_{I}^{x}$, where $c=1$ is a constant, $I_{P L}$ is the output image, $I_{I}$ is the input NIR image and $x=0.7$.

2. Threshold $I_{P L}$ with a value of 0.1 . The resulting binary image, $I_{B W}$, has the pupil and eyelashes denoted by 1 .

3. Find the contour of the sclera region as segmented in Section 5.1, $I_{S C H}$.

4. Use Hough transform for line detection. Select and remove the highest peak corresponding to the longest line.

5 . Fit an ellipse to the remaining sclera contour points, $E\left(a, b,\left(x_{0}, y_{0}\right), \theta\right)$, where $a, b,\left(x_{0}, y_{0}\right)$ and $\theta$ correspond to the length of the semi-major axes, length of the semi-minor axes, the center of the ellipse, and its orientation, respec-

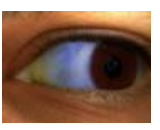

(a)

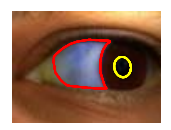

(b)

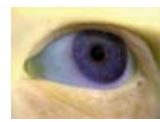

(c)

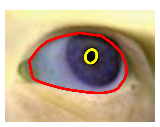

(d)
Figure 8. Pupil segmentation. a) Eye with dark color iris. b) Segmented pupil for dark color iris. c) Eye with light color iris. d) Segmented pupil for light color iris

tively. Define an elliptical mask (to detect the pupil region) to extract the pixels located within the ellipse.

6. Impose the ellipse mask on the binary image $I_{B W}$ obtained in step 2. The result is a binary image that will contain the pupil, and possibly eyelashes, as logical 1 pixels, $I_{P}$.

7. Count the number of connected objects $\mathrm{N}$ in $I_{P}$. If $N>1$, through an iterative process, decrease the ellipse's semi-major and semi-minor axis (by $2 \%$ ) and construct new elliptical masks that when imposed on the binary image $I_{B W}$ will render a smaller value for $\mathrm{N}$. The connected object for $N=1$ will correspond to the location of the pupil.

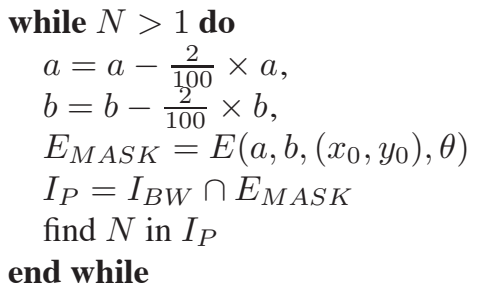

8. Fit a new ellipse $E$ to the dilated region corresponding to the location of the pupil. Compute $I_{P}=I_{B W} \cap E_{M A S K}$. Even if low intensity regions in the iris are inadvertently selected, the pupil region has by far the largest area among all connected objects.

9. Fit an ellipse to the pixels pertaining to the pupil region to find the pupil mask, $P_{M A S K}$. Resize the pupil mask to the original NIR image size.

The procedure described above is applied to all the images regardless of the color of the iris. For 15 images, the algorithm failed to correctly segment the pupil.

\subsection{Sclera segmentation}

As mentioned in Section 5.1, for light color iris images, further segmentation of the iris is needed. The criteria to finalize the segmentation of the sclera is given by the intersection of the pupil region, $P_{M A S K}$, as found in Section 5.2 and the sclera region, $S_{M A S K}$, as found in Section 5.1.

\subsubsection{Iris segmentation}

We use the k-means clustering algorithm $(\mathrm{k}=2)$ to segment the iris. The algorithm uses the pixels contained within the sclera mask $S_{M A S K}$ (Figure 9(e)) as its input. Each pixel is viewed as a three-dimensional entity consisting of 


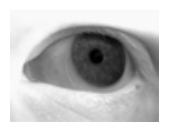

(a)

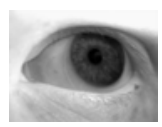

(b)

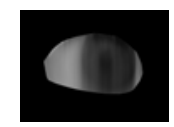

(c)

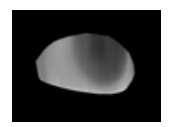

(d)

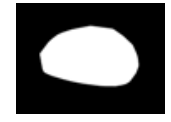

(e)

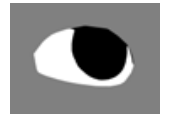

(f)

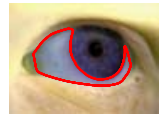

(g)
Figure 9. Iris segmentation for light color iris. a) NIR component. b) Red component. c) Proportion of sclera in north direction $p_{\uparrow}(x, y)$. d) Proportion of sclera in south direction $p_{\downarrow}(x, y)$. e) Convex hull of the sclera $I_{S C H}$. f) K-means output. g) Segmented sclera

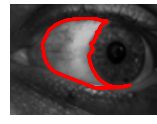

(a)

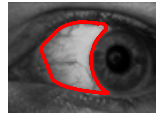

(b)

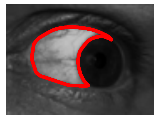

(c)

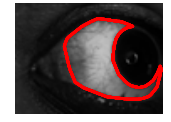

(d)
Figure 10. Contour of the segmented sclera imposed on the green component. a) and b) light color iris. c) and d) dark color iris

the intensity value of the NIR component (Figure 9(a)), intensity value of the red component (Figure 9(b)), and the proportion of sclera in the north $p_{\uparrow}(x, y)$ and south directions $p_{\downarrow}(x, y)$ [7], as assessed in the red component (Figure 9 (c)(d)). The value of $p(x, y)$ is set to 0 for all the pixels outside the sclera region. For a pixel $(x, y)$ inside the sclera region, the proportion of sclera in the north direction, $p_{\uparrow}(x, y)$, is computed as the mean of all the pixels of column $y$ above $(x, y)$, and the proportion of sclera in the south direction, $p_{\downarrow}(x, y)$, is computed as the mean of all the pixels of column $y$ below $(x, y)$. Euclidean distances between the origin of the coordinate system and the centroid of each cluster are computed in order to determine the label of the two clusters (the label can be 'sclera' or 'iris'). The largest distance is associated with the sclera cluster; this is the white region in Figure 9(f). The smallest distance is associated with the iris cluster; this is the black region in Figure 9(f). Two binary images, a mask for the sclera region (Figure 9(f), pixel value 1) and a mask for the iris region (Figure 9(f), pixel value 0), represent the output. On examining the two binary masks, we observe that in some images, the k-means algorithm erroneously labels portion of the sclera as being the iris (mainly the corners of the sclera that are less illuminated and have lower intensity values). To address this issue, we find that region in the iris mask that overlaps with the pupil mask and subtract it from the original sclera region $I_{S C H}$.

\section{Enhancement of blood vessels observed in the sclera region}

To improve segmentation of the blood vessel patterns, the segmented sclera image is pre-processed in two con- secutive steps: image enhancement followed by line enhancement. In the first step, the RGB image is converted to the $\mathrm{L}^{*} \mathrm{a} * \mathrm{~b}$ color space. Contrast-limited adaptive histogram equalization (CLAHE) is applied to the luminance component $\mathrm{L}^{*}$. The algorithm divides the entire image into smaller square tiles. Each tile is enhanced using histogram equalization. This induces artificial boundaries between tiles that are removed using bilinear interpolation. The enhanced $\mathrm{L}^{*} \mathrm{a} * \mathrm{~b}$ image is then converted back to the RGB color space. An examination of the three components of the RGB image suggests that the green component has the best contrast between the blood vessels and the background. In the second step, a selective enhancement filter for lines as described in [8] is applied to the green component. The enhancement filter for lines, and implicitly for blood vessels, is described by the equation:

$$
I_{\text {line }}\left(\lambda_{1}, \lambda_{2}\right)=\left\{\begin{array}{rll}
\left|\lambda_{1}\right|-\left|\lambda_{2}\right|, & \text { if } & \lambda_{1}<0 \\
0, & \text { if } & \lambda_{1} \geq 0
\end{array}\right.
$$

where, $\lambda_{1}$ and $\lambda_{2}$ (with $\left|\lambda_{1}\right|>\left|\lambda_{2}\right|$ ) are the two eigenvalues of the Hessian matrix of each pixel and computed as follows: $\lambda_{1}=K+\sqrt{\left(K^{2}-Q^{2}\right)}, \lambda_{2}=K-\sqrt{\left(K^{2}-Q^{2}\right)}$, where $K=\left(I_{x x}+I_{y y}\right) / 2, Q=\sqrt{\left(I_{x x} * I_{y y}-I_{x y} * I_{y x}\right)}$, $I_{x x}, I_{y y}, I_{x y}$ and $I_{y x}$ represent the second-order derivatives in $\mathrm{x}$ and $\mathrm{y}$ directions. The algorithm for blood vessels enhancement is based on [8]:

1. Determine the minimum $\left(d_{\min }\right)$ and maximum $\left(d_{\max }\right)$ diameter of the blood vessels.

2. Consider $\mathrm{N}(=5)$ multiple $2 \mathrm{D}$ Gaussian distributions with standard deviation, $\sigma$, within the interval $\left[d_{\min } / 4\right.$, $\left.d_{\max } / 4\right] . \sigma=[0.25,0.5,1,2,3]$

3. Convolve each Gaussian distribution with the original image.

4. Compute the two eigenvalues for each pixel, for each of the $\mathrm{N}$ convolved images.

5. Using the eigenvalues, compute $I_{\text {line }}$.

6 . Multiply each pixel with the square of the corresponding Gaussian standard deviation: $I_{\text {line }} * \sigma^{2}$.

7. Compute the maximum value at each pixel based on the $\mathrm{N}$ outputs: $I_{\text {out }}=\operatorname{argmax}\left(I_{\text {line }} * \sigma^{2}\right)$.

\section{Registration}

Image registration is the process of finding a transformation that aligns one image with another. The method used here, described in [6], models a local affine and a global smooth transformation. It also accounts for contrast and brightness variations between the two images that are to be registered. The registration between two images, the source $f(x, y, t)$ and the target $f(\hat{x}, \hat{y}, t-1)$, is modeled by the transformation $\vec{m}=\left(m_{1}, m_{2}, m_{3}, m_{4}, m_{5}, m_{6}, m_{7}, m_{8}\right)$ : 


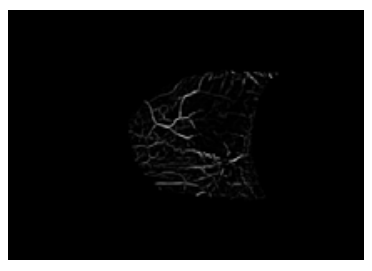

$(n)$

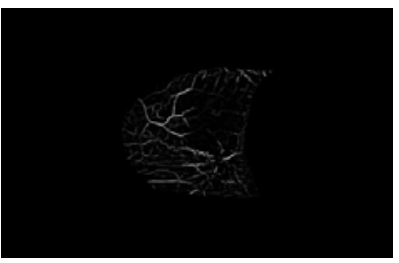

(h)

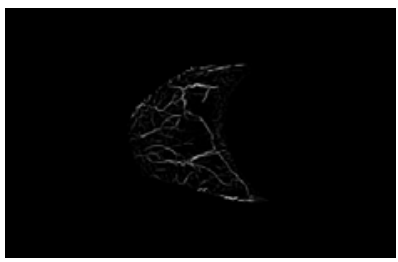

(c)

Figure 13. Matching. a) user1-image1. b) user1-image2. c) user2image1. Match scores: a) with b) $=0.94952$; a) with c) $=0.25611$

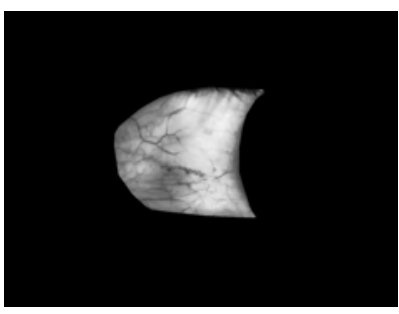

(a)

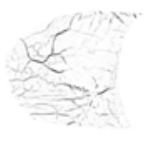

(b)
Figure 11. Blood vessel enhancement. (a) Green component of the segmented sclera. (b) The complement of enhanced blood vessels
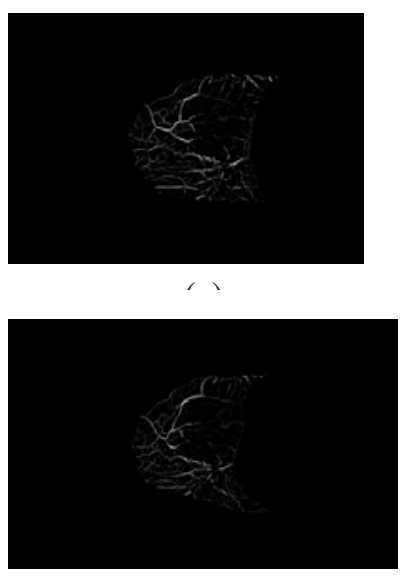

(c)
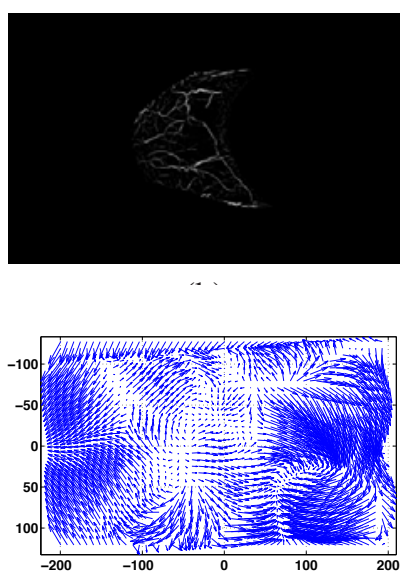

(d)
Figure 12. Image registration. a) Source image. b) Target image. c) Registered source. d) Flow image depicting the warping process

$m_{7} f(x, y, t)+m_{8}=f\left(m_{1} x+m_{2} y+m_{5}, m_{3} x+m_{4} y+\right.$ $\left.m_{6}, t-1\right)$, where $m_{1}, m_{2}, m_{3}$, and $m_{4}$ are the linear affine parameters, $m_{5}, m_{6}$ are the translation parameters, and $m_{7}, m_{8}$ are the contrast and brightness parameters. A multiscale approach is employed by using a Gaussian pyramid to downsample the images to be registered. From a coarse-tofine level, the transformation $\vec{m}$ is determined globally at each level, and then locally, and the estimated parameters are used to warp the source image. Figure 12 shows results of the registration on two pre-processed sclera images. Using the linear affine parameters $m_{1}, m_{2}, m_{3}$, and $m_{4}$, and the translation parameters $m_{5}, m_{6}$, the sclera mask of the source image is also registered.

\section{Matching}

The similarity between two sclera images is assessed using cross-correlation between regions of the sclera within the overlap region of the two sclera masks as shown in Figure 13. To generate genuine scores, this cross-correlation is performed between pairs of images for each subject; to generate the impostor scores, cross-correlation is performed between the first images of each subject pair. Results displayed in Figure 14 using Receiver Operating Characteristic (ROC) curves indicate an EER of 0.0 for left-eye-lookingleft, an EER of 0.3247 for left-eye-looking-right, an EER of 0.5128 for right-eye-looking-left and an EER of 0.9776 for right-eye-looking-right. Initial analysis indicates that an EER of 0.0 for left-eye-looking-left was obtained because of the small number of subjects and the constraints imposed on data acquisition. The results also suggest that performance is improved considerably (see [2] for comparison) if (a) the variations in the pose and the amount of specular reflection are reduced, and (b) the images are of higher resolution/quality.

To demonstrate the necessity of using the proposed pre-processing techniques, the d-prime value ${ }^{8}$ was computed for 4 scenarios on a small subset of 15 subjects (images 1, 4, 8 from the sequence left-eye-looking-left): registration and correlation (d-prime $=2.8058$ ); image enhancement, registration, and correlation (d-prime $=$ 3.5664); line enhancement, registration, and correlation (d-prime $=8.2698)$; image enhancement, line enhancement, registration, and correlation $(\mathrm{d}$-prime $=8.1965)$. The last two scenarios resulted in the best d-prime values thereby suggesting the importance of using the pre-processing techniques.

\section{Summary and Future work}

In this paper we designed segmentation, enhancement and matching routines for processing the conjunctival vasculature of multispectral eye images pertaining to 49 subjects. The images used in this work were acquired under

\footnotetext{
${ }^{8}$ The d-prime value measures the separation between the genuine and impostor score distributions. A higher value typically suggests a better performance
} 


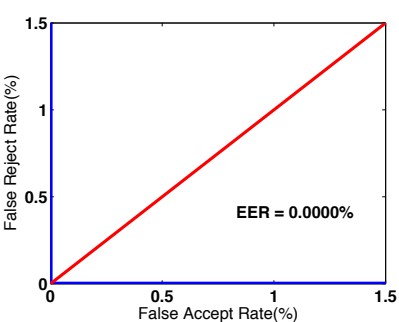

(a)

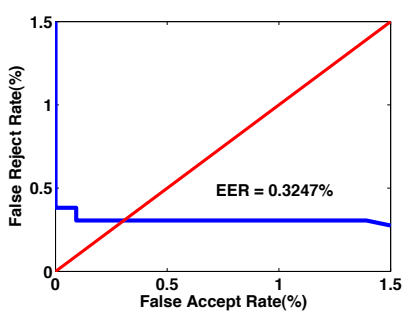

(c)

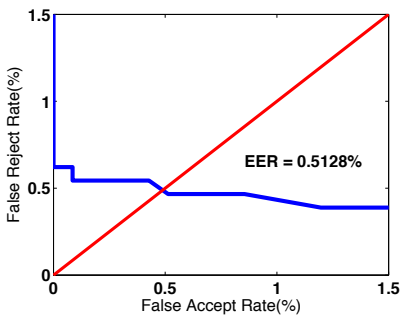

(e)

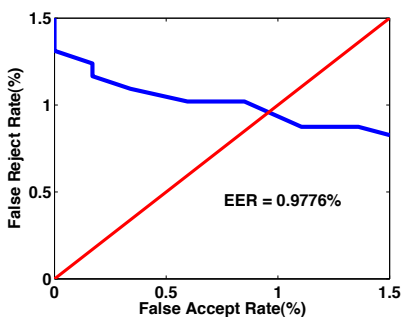

(g)

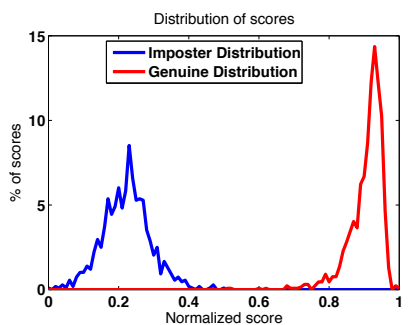

(b)

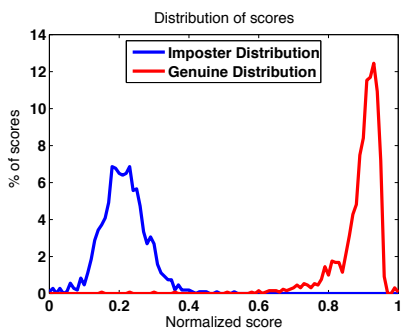

(d)

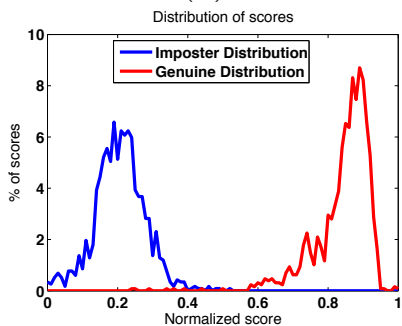

(f)

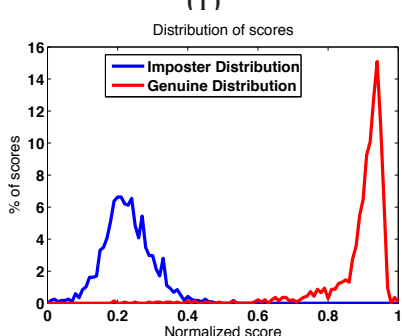

(h)
Figure 14. ROC and Normalized scores. a) and b) Left eye, looking left. c) and d) Left eye, looking right. e) and f) Right eye, looking left. g) and h) Right eye, looking right. The excellent performance can be attributed to the constraints imposed during data acquisition. In operational scenarios, conjunctival vasculature may be more suited as a soft biometric

several constraints to limit specular reflections and variations in pose as much as possible. The use of multispectral imagery will be beneficial when combining iris patterns with conjunctival vasculature. Since iris patterns are better resolved in the near-infrared spectrum and the conjuncti- val patterns are better resolved in the visible spectrum, we are looking at ways to combine the conjunctival vasculature with the iris for enhanced biometric recognition. Further, we are designing schemes to address the problem of pose variation that can impact the performance of this soft biometric.

\section{References}

[1] K. W. Bowyer, K. Hollingsworth, and P. J. Flynn. Image understanding for iris biometrics: A survey. Computer Vision and Image Understanding, 110(2):281 - 307, May 2008.

[2] S. Crihalmeanu, A. Ross, and R. Derakhshani. Enhancement and registration schemes for matching conjunctival vasculature. Proc. of International Conference on Biometrics, Alghero, Italy, pages 1240-1249, June 2-5 2009.

[3] J. Daugman. How iris recognition works. IEEE Transactions on Circuit and Systems for Video Technology, 14(1):21-30, 2004.

[4] R. Derakhshani, A. Ross, and S. Crihalmeanu. A new biometric modality based on conjunctival vasculature. Proceedings of Artificial Neural Networks in Engineering, St.Louis, MO, November 2006.

[5] R. C. Gonzales and R. E. Woods. Digital image processing. 2001. Prentice-Hall Inc., 2nd edition.

[6] S. Periaswamy and H. Farid. Elastic registration in the presence of intensity variations. IEEE Transactions on Medical Imaging, 22(7):865-874, 2003.

[7] H. Proenca. Iris recognition: On the segmentation of degraded images acquired in the visible wavelength. IEEE Transaction on Pattern Analysis and Machine Intelligence, 32(8):1502-1516, August 2010.

[8] L. Qiang, S. Shusuke, and D. Kunio. Selective enhancement filters for nodules, vessels, and airway walls in two or three dimentional CT scans. Medical Physics, 30(8), 2003.

[9] A. Ross. Iris recognition: The path forward. IEEE Computer, pages 30-35, February 2010.

[10] I. W. Selenick. The double density DWT. pages 39-69, 2001. Chapter in "Wavelets in Signal And Image Analysis: from Theory to Practice" (Eds: A. Petrosian and F. G. Meyer).

[11] I. W. Selenick. A new complex-directional wavelet transform and its application to image denoising. IEEE International Conference on Image Processing, 3:573-576, 2002.

[12] I. W. Selenick. The double-density dual-tree DWT. IEEE Transactions on Signal Processing, 52(5):1304-1314, 2004.

[13] C. J. Tucker. Red and photographic infrared linear combinations for monitoring vegetation. Remote Sensing Environment, 8:127-150, 1979. 